\title{
A Group Awareness System for Blinds in Web Co- Authoring Environment
}

\author{
Waqar M. M., Aslam Muhammad, Talha Waheed \\ Dept. of CS \& E., U.E.T. \\ Lahore, Pakistan \\ mirzamwaqar@hotmail.com, maslam@uet.edu.pk, \\ twaheed@gmail.com
}

\author{
Martinez Enriquez A. M. \\ Department of Computer Science \\ CINVESTAV, D.F., Mexico \\ ammartin@cinvestav.mx
}

\begin{abstract}
Generally, it is seen that the disable people most commonly blinds in our society are not encouraged to work with normal community due to lack of vision, communication, and understandings. Fortunately, this is no longer a big issue. By use of modern technology and tools we can develop flexible environments in which blinds work collaboratively with normal ones and share their knowledge with each other to produce a robust and worthy task. In this research, we solve the issue by proposing a platform for different categories of people (fully/partially impaired and normal). The locally/remotely distributed participants work together (online or offline) in a Web based co-authoring environment to produce a shared document. The system presents a simplified design of mechanisms to share, modify, and update document and their resources in a consistent and controlled way such that each group member can easily able to know the updates and be well aware about the current state of the common object. The main goal of the research is to make the handicap persons self-reliant, confident, and independent so that they can play their equal part in every group activity of the society. The approach is evaluated by means of experiments conducted with blinds and normal authors in a distributed environment.
\end{abstract}

Keywords-CSCW, Web Co-authoring, Group Activity, Group Awareness, Distributed Co-operative Work.

\section{INTRODUCTION}

According to World Health Organization (WHO), the total number of visually impaired people worldwide are around 39 million [13]. So the blind people play a major role in our society and they need to interact and communicate with each other and normal peers. For this, they need to use technology but unfortunately the blind people are not able to see and read the instruction available in visual format as the existing technology is rarely designed to be used by the fully or partially impaired persons. Now, this is no longer a big issue because the new gadgets offer good alternatives to facilitate disable people. The blind use the assistive technology tools, like screen readers/speech synthesizer [17] [18], voice recognition, and braille translation tools [14] [15], etc., to interact with the computer system and devices. Also, new technologies deal with a powerful mechanism of interaction among individuals: the collaborative writing. This mechanism allows the diversity of knowledge and skills and helps individuals who have difficulty to formalize certain knowledge or build solution alone. Thus, problem can be better solved by a group of individuals working collaboratively, than by a single individual [2].

The Computer Supported Cooperative Work, CSCW is related to the technology whose goal is to support groups of people collaborating to reach a common goal or perform a common task [16]. The special category of people, like blind, could not equally participate in a collaborative environment because these tools are not in general designed to be effectively used by them and also the notification and awareness functions offered in these systems do not have any extended features especially deliberate to them. There is a lack of awareness of group activities happening in that environment for these kind of special persons. Thus, to produce collaboration in special people, there must be a need of an artificially complemented environment for different categories of people with different awareness facilities like special kind of notification, alert beep, text to speech tools that remain external to the cooperative production functionalities.

The main goal of the research is to make the handicap persons self-reliant, confident, and independent so that they can play their equal part in every group activity of the society. This objective is achieved by developing a platform with a notifications system. The platform provides an interface to special people to actively participate in the group activities and fulfill a common goal and a well-structured notification/ information system is embedded in this platform to provide good awareness about the events occurred in shared space. We have provided good communication service to frequently exchange information between disables and normal. Efficient functionalities are provided to deduce knowledge about the contributions produced by others to coordinate their activities. Awareness supports dedicated to offer functionalities, tools and interfaces that fulfill the requirements of special people.

Section 2 discusses the related work. Section 3 is devoted to the questioner that has been conduct during this research and explains the results obtained from it. The architecture of proposed framework and the comprehensive overview of its each component has enlightened in Section 4. Section 5 is the conclusion of this research and also gives some future directions.

\section{LITERATURE REVIEW}

In group work environment, we can gain efficiency in time and improve the quality of work while solving the complex problems and as a result we get a better final solution. The group members of the team work cooperatively and 
coordinately to achieve the high productivity and quality [1]. So, all these aspects are integrated while we discuss about coauthoring platform where different team members work on a single document to make it effective and worthy. Normally in co-authoring environment the shared document is divided into different sections and each section is owned by one of coauthor. The ownership may change time to time depends on the interest and requirements of the co-author and documents. In a cooperative work, there should be a need of group awareness and coordination support that can meet the quality of cooperation. The group awareness is related to the information about group members who are participating in the coauthoring, the action they performed during group activities and the effects of ones activity with others' work. [2]. The awareness becomes a challenge when there is an author that is blind.

The collaborator is a CSCW system that manages synchronous as well as asynchronous ways of communication and is used in big organizations [7]. Some of the examples of such systems include SASSE [4] and JAMM [5]. While implementing these systems, certain issues have had to be addressed. The one of the major issue is inconsistency. In collaborative work normally a document is replicated on different site or places which may cause of inconsistency. In solution of this problem Y. Yang et al. [6] presented a consistency model. In some system, instead of using general document editor, they preferred to use specific application models for a particular use. M. Locasto et al. presented a system named Clay [7] which gives a collaborative environment for the developers geographically located at different places to work to gather synchronously. Another collaborative working platform is created by Qingzhang et al. [8] which is particularly based on XML documents.

Ho, Leong et al. [12] presented a framework in which the shared document written by distributed authors is converted into XML format. The advantage of using XML format to store content of any general document is to use the attributes of XML in document processes. As an example, the fragment nature of XML documents is used to control the access and locking the content of the documents and used to manage resources. WoTel [9] is a prototype used to conduct audio/video conference while working on a shared document. A multimedia collaboration system is integrated to allow users to do group communication and share the ideas with each other. Hodel et al. [10] presented an architecture named TeNDeX used to do synchronous document editing. Unlike of other conventional co-authoring platform, they used database to save the content of the document. One of another application of synchronous collaboration is explained by Joeris et al [11], who used this technology to support engineering domain. [16] is a collaborative writing platform for writing mathematical expressions. The responsibility of the system is to evaluate the written mathematical formula and provide the suggestions similar to the written one and reuse the old one. It also informs the author about the possible expert writer currently available during the writing of mathematical expression.

On the other hand, if we discuss about the tools and technology used by blind while writing any documents then the first one that needs to be discussed is text to speech convertor. Text to speech conversion system has been developed in
Windows, Mac and, other operating systems for those who learn things easily by listening instead of reading; this feature provides voice output of text. This technique is applied to system design for totally blind persons. The review of Apple's Text to Speech Software "Voice Over" made by Jay Leventhal [17] shows that some features are missing, e.g. An Online Help handbook is not accessible to the person who is quite new to Apple keyboard. Another shortcoming is the integration of the software with the Web browser Safari used in Mac X. So, in the words of Leventhal, the first version of Voice Over was disappointing one. However, the user friendliness of Apple Mac can be enhanced by close contact training [18], in which the Text-to-Speech conversion system is implemented in the collaborative suthoring environment to assist the visually impaired persons and minimizes the need of the sighted persons as a support. Thus, they are able to produce an excellent document by the group work.

Similarly, another important tool needed by blinds during document reading/printing is text to Braille translators. There are many text to Braille transliteration tools are available for different languages. The most popular systems used for English language are Duxbury [www.duxburysystems.com], JAWS [http://www.freedomscientific.com/fs_products/software_jaws. asp], and WinBraille [www.braille.se/downloads/ winbraille.htm]. For non-English language, a toolset named Sparsha [14] is available to transliterate Indian language into Braille. The unique feature of this system are transliterating the mathematical symbols and tactile graphics to braille. Apart from this, Kalra et al. [15] developed a system which works as a Braille writing tutor. The prototype tutor system uses an eslate device to capture a student's action and tries to develop the Braille writing skills.

Hence, from the above discussion it is concluded that there are many co-authoring framework and platform exists. Also there are many tools available for the blinds during writing and reading documents, but unfortunately these tools are developed for individual tasks. Similarly in co-authoring environments there is not any extra features or tools that can help blind to work in group environment. So, no such a platform is currently available which can provide a collaborative and cooperative interface for the blind. This lack motivated us to propose a platform for blinds and encourage them to work along with normal peers to share their ideas and knowledge.

\section{NEED OF WEB-COAUTHRING FOR THE BLINDS}

A survey was conducted during this research. The objective of the survey was to know that either the blinds had done any group activity or not and if yes then what kind of deficiencies and problems they faced during that group work. For this purpose, we had made a questioner and distributed it among individual blinds and special educational institutes located globally via email. We got almost 150 responses from different blind persons. The results obtained from this survey were very promising and encouraged us to make a system that can help the blinds to work in a group environment along with the normal on a single goal.

The questioner is divided into two sections. The Section-I includes the general questions to ask them about their interaction with computer system and one major question about either they had ever done any group activity or not. The most 
of the questions have options yes/no so that it can be easy for blinds to answer. Some major questions included in Section-I are: Do you think modern technology helps to solve the problems or improve your working performance? $88 \%$ said yes. Another question is: Do you like to perform group activities? Yes is answered by $64 \%$. Which communication mode you preferred when you are at different places. $60 \%$ replied internet like social networking software and 33\% answered phone. This means they like to spend their time on Web and feel comfortable using Web applications. Similarly a question asked: If you have a common goal, which mode of group work will you prefer? And the options are 1) shared document 2) Individually and Merge Later 3) Both according to need. The $80 \%$ of the people selected option 2 . The main reason of this answer is that they do not know any particular tools which are especially designed for blind to perform group activity. Normally they work on a shared document and merge their documents manually which may cause inconsistency and there is a huge chance of duplicate content. Also, at the time of merging document they know about the updates and changes made by all authors that may causes the repetition and conflicts of the contents.

Whereas, the section-II is particularly for those persons who had done a group activities and their feedback about that activity. Here, are the list of questions included in section-II of the questioner. These questions is asked to answer by only those persons who have answered "YES" to question Have you ever done any group activity? of section-I.

TABLE I. RESUlTS OF SECTION-II OF THE QUETIONEER

\begin{tabular}{|c|c|c|c|c|}
\hline Sr. & Question & Option-1 & Option-2 & Option-3 \\
\hline \multirow[t]{2}{*}{1.} & \multirow{2}{*}{$\begin{array}{l}\text { When working in a group, does you know abilities of each } \\
\text { other? }\end{array}$} & Yes & NO & Don't Know \\
\hline & & $36 \%$ & $64 \%$ & $0 \%$ \\
\hline \multirow[t]{2}{*}{2.} & \multirow{2}{*}{$\begin{array}{l}\text { How do you share your documents when you are working on } \\
\text { common goat but located at different places? }\end{array}$} & Via Mail & Shared Drive & Other \\
\hline & & $84 \%$ & $12 \%$ & $4 \%$ \\
\hline \multirow[t]{2}{*}{3.} & \multirow[t]{2}{*}{$\begin{array}{l}\text { How do you get aware about the changes and updates your } \\
\text { co-author make in document? }\end{array}$} & Manually & $\begin{array}{l}\text { Notification } \\
\text { System }\end{array}$ & Other \\
\hline & & $91 \%$ & $6 \%$ & $3 \%$ \\
\hline \multirow[t]{2}{*}{4.} & \multirow[t]{2}{*}{$\begin{array}{l}\text { For synching all group member's document to make global } \\
\text { document, what method do you use? }\end{array}$} & Manually & $\begin{array}{l}\text { Document } \\
\text { Merging } \\
\text { Software } \\
\end{array}$ & Other \\
\hline & & $93 \%$ & $0 \%$ & $7 \%$ \\
\hline \multirow[t]{2}{*}{5.} & \multirow{2}{*}{$\begin{array}{l}\text { It is easy for you to do a group activity, when the authors are } \\
\text { in same location. }\end{array}$} & Yes & $\mathrm{NO}$ & Don't Know \\
\hline & & $96 \%$ & $2 \%$ & $2 \%$ \\
\hline \multirow[t]{2}{*}{6.} & \multirow{2}{*}{$\begin{array}{l}\text { It is easy for you to do a group activity, when the authors are } \\
\text { in distributive environment. }\end{array}$} & Yes & NO & Don't Know \\
\hline & & $43 \%$ & $48 \%$ & $9 \%$ \\
\hline \multirow[t]{2}{*}{7.} & \multirow[t]{2}{*}{ Are you encouraged to work a group activity with normal? } & Yes & NO & Don't Know \\
\hline & & $25 \%$ & $75 \%$ & $0 \%$ \\
\hline \multirow[t]{2}{*}{8.} & \multirow{2}{*}{$\begin{array}{l}\text { Do you feel comfortable while working any group activity } \\
\text { with normal? }\end{array}$} & Yes & NO & Don't Know \\
\hline & & $35 \%$ & $65 \%$ & $0 \%$ \\
\hline \multirow[t]{2}{*}{9.} & \multirow{2}{*}{$\begin{array}{l}\text { Have you ever used any co-authoring/CSCW framework } \\
\text { earlier? }\end{array}$} & Yes & NO & Don't Know \\
\hline & & $6 \%$ & $82 \%$ & $2 \%$ \\
\hline \multirow[t]{2}{*}{10.} & \multirow[t]{2}{*}{ Did that platform have any special features for blinds? } & Yes & NO & Don't Know \\
\hline & & $0 \%$ & $6 \%$ & $84 \%$ \\
\hline \multirow[t]{2}{*}{11.} & \multirow[t]{2}{*}{$\begin{array}{l}\text { Will you prefer to use any CSCW platform with special } \\
\text { features for blind or use your ordinary techniques? }\end{array}$} & CSCW Platform & $\begin{array}{l}\text { Ordinary } \\
\text { System }\end{array}$ & Don't Know \\
\hline & & $74 \%$ & $8 \%$ & $18 \%$ \\
\hline
\end{tabular}

From the above results it was concluded that the blinds like to do group activities but they do not have any preferable resources or tools that helps them to work comfortably in these activities. They are using the ordinary techniques to fulfill their goal. They do not have any special kind of features and tools available in any group activity platform. Most of them are unfamiliar with CSCW platform as they have never seen this kind of software. The one reason of not using these platform is they do not native to blinds. So, we were in a better position to develop a system that have more intelligent and needful for blinds.

\section{WEB CO-AUTHORING FRAMWORK FOR THE BLIND}

When anyone working in a cooperative work environment then he/she must know about the progress and progression of the document, the actions performed by the co-author, etc. To 
improve the contribution of individual authors, they must well aware about the activities of the whole group, the awareness is an important attribute of collaborative activities [1]. If we say awareness it means the individual should know about group activities which have been performed in past and present. Also the members must have knowledge about the group and the current state of the group [2]. By use of awareness information, a context of an individual work is formed by comparing it with the work of rest of the group. By use of this context, the users synchronize their work and coordinate with each other. This context is a necessary element for an individual for measuring his work with respect to the goal and progress of group [3]. The objective of exchanging information is to inform the coauthors about the activities performed by an individual.

The Web Co-authoring Framework for the Blind (WCFB) presents a web based cooperative authoring platform to work on a single goal. The system is developed by use of advance technology like HTTP, XML, SMIL, etc. and an open source Web authoring library Amaya [19] as a kernel of this system.
The architecture of the proposed platform is based on a three layer distributed model, a) Amaya Thot library [19], b) Intelligent Interface for Blind's Awareness (IIBA) framework, and c) HTTP web technology, that integrates key components to provide support for cooperative authoring on the Web for blinds(see Fig. 1). The upper layer shows that cooperative applications are built on the top of it. Based on their functional requirements, these applications use the features provided by this middleware. In middleware, we build a group awareness inference engine (IIBA) to control the awareness functions and feature for the authors especially blind authors. In the bottom layer, we see a set of basic entities for supporting cooperative authoring interface. They are managed by extensible awareness features located in the middle layer. Fig. 1 also shows that a further decomposition of each layer brings up individual components for managing each one of the following entities: authors, documents, and their resources, sessions, projects, events, applications, and group awareness.

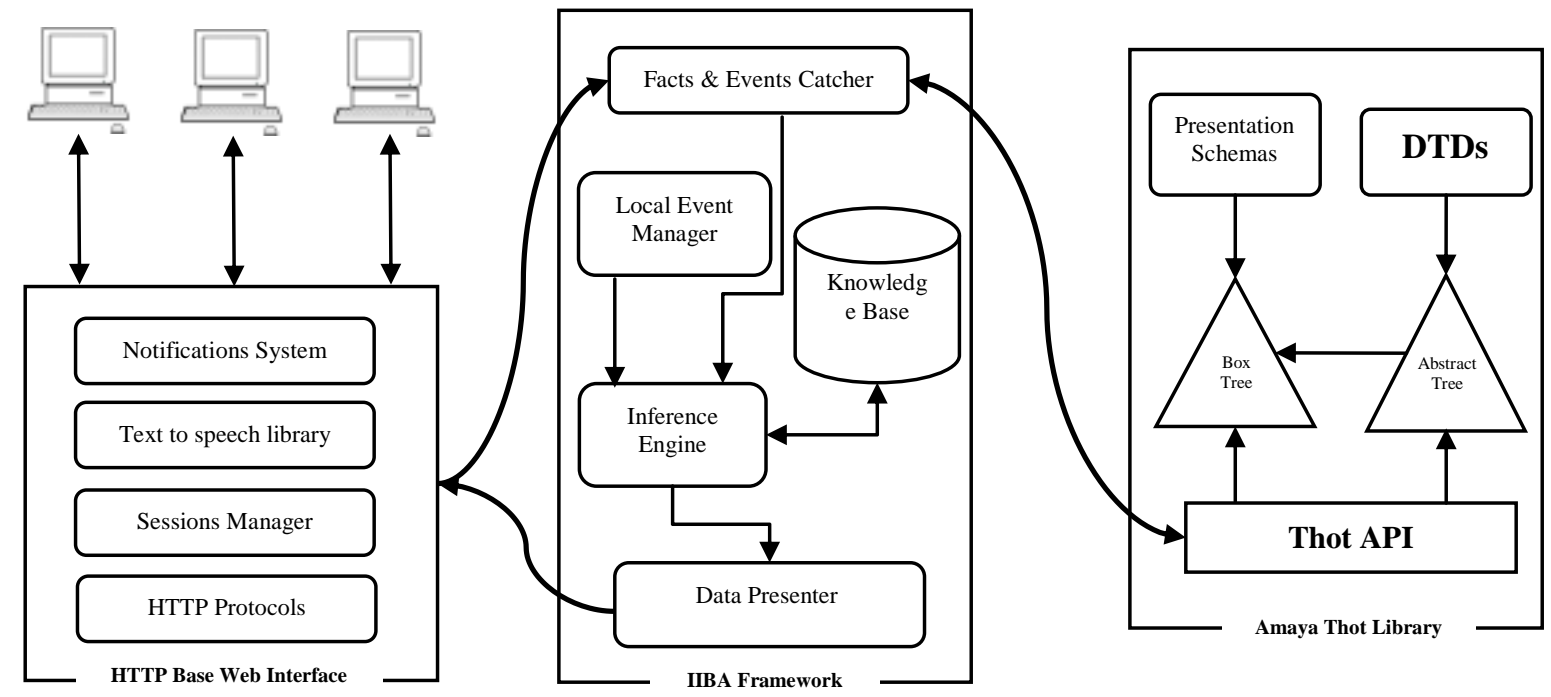

Fig. 1. Core Architecture of WCFB

\section{A. Amaya's Thot Library}

Amya is an open source library and in its software architecture there are available several APIs and functions which can be used to update and enhance the functionality with small changes [19]. Amaya uses the Thot tool kit for handling the shared documents in a structured way. Thot is the combination of libraries the gives the functionalities for manipulating the document. It keeps the document in a structured way by separating the content, structure and presentation. Thot internally represent the document in the form of tree, called the Abstract tree, whose elements are headings, paragraphs, lists, phrases etc. The attributes are associated with these elements to present the advance information about the element's role in the documents structure. DTDs contains set of rules that maintains the document in a logical structure. By use of these rules, the elements and attributes are assembled to make a structured document and also state the association of any attributes with its related elements. A set of views to present the document image is composed in Presentation Schema. A view is a combination of presentation rules that define the elements and attributes in corresponding structure schema to be proposed to the user. The Thot uses the presentation schema and abstract tree to format the document and produces an intermediate structure the Box Tree, which is used to present the views. Finally, the Thot API provides and interface that is used to communicate with the outer environment of Amaya framework. We have several functions used to send and receive the document elements and attributes and hooks to manipulate the document.

\section{B. Intelligent Interface for Blind's Awareness (IIBA) Framework}

The main focus of this research is to design and implement the middle layer of the proposed system named an Intelligent Interface for Blind's Awareness (IIBA), which enhance the awareness for blind authors by which they can work easily and 
comfortably in shared environment. This layer is work as a bridge between kernel and interface layer. The key component of IIBA are: Facts and Event Catcher who captures different action and events like opening and closing sessions of the shared documents, the updated performed during documents editing, data consultation, user's actions e.g. who did perform an action, from where did he do this, what was the action, etc. The system establish a dedicated storage space to save these events to deliver to consumer application. The Local Event Manager receives the events generated by local application and authors. The events are classified following their arrival date in the system. All events are stored in order list implemented as circular buffer. All these stored local event are later sent to other users that take part in cooperative environment so that they aware about all the cooperative events related with shared document. All these fetched events either local or remote are transferred to Inference Engine who takes them as input, analyze them by use of rules stored in Knowledge Base and performed corresponding actions. The rules in the Knowledge Base are written in first order predicate logic. The rules are consists of two parts the premise and action. All the rules are well defined and all type of lexical, syntactical and semantically errors are removed. As an example when a coauthor opens a shared document then a notification is sent to all other authors present in that session. For this the following rule is triggered.

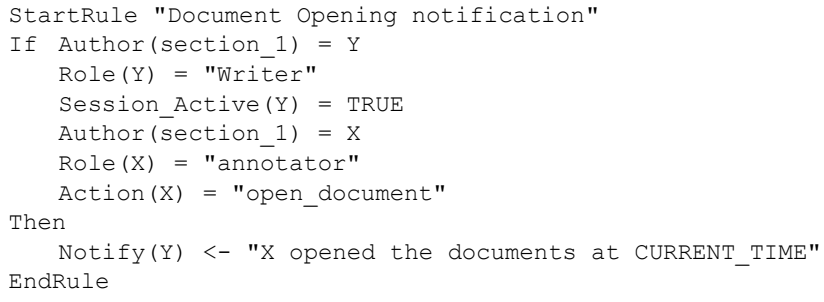

The semantic of the condition of this rule is as follows:

- Author(section_1) $=\mathrm{Y}$, defines the set of $\mathrm{Y}$ of coauthors of section 1 of the document.

- $\operatorname{Role}(\mathrm{Y})=$ "Writer", checks that is the role of the author is Writer

- Session_Active(Y) = TRUE, checks is the author $\mathrm{Y}$ is current logged in to the environment.

- $\operatorname{Action}(\mathrm{X})=$ "open_document", defines the coauthor $\mathrm{X}$ is opening the shared document.

The semantic of the actions of this rule is:

- $\operatorname{Notify}(\mathrm{Y})$ <- "X opened the documents at CURRENT_TIME", notify users $\mathrm{Y}$ that user $\mathrm{X}$ has opened the document at particular time.

Similarly when any co-author closes the opened document then to send notification to active coauthors the following rule is triggered.

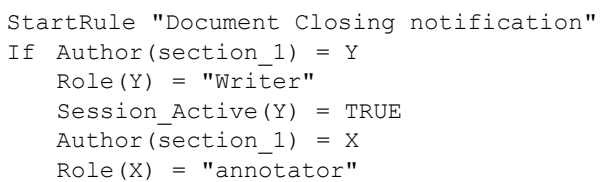

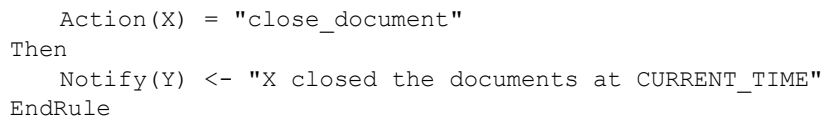

Whenever, a user starts his session, the inference engine automatically starts and continues until the end of his session to inform him about the activities of other co-authors and vice versa. The working of Data Presenter is to gather the information from inference engine, make it presentable and transmit it to HTTP host who provided an interface to the authors to use application.

\section{HTTP Based Web Interface}

The responsibility of this layer is to provide an interactive interface to the authors in which they can well aware about shared environment. Special tools and libraries enhance the user interface for blind authors. HTTP is set of rules that are used to send and receive data and information from server to client and vise versa. Whenever, a user starts his session then indirectly he starts using HTTP. Session Manager uniquely identifies the authors to control their access on shared document and also protect them from unauthorized access. It also keep record of author's starting and leaving of shared environment. It is important because when multiple users work in shared environment then they are interested to know that when a coauthor started the cooperative session and when he left the session. Notification System informs authors of what they need to know as a result of other action of other coauthors. It notify through different type of beeps single for different actions because blind can only listen but cannot see. Like an opening door audio is rung when any co-author starts session, a small beep represents some modification done in the document. A long beep is to give update about the change of role of any co-author. Different hot keys are informed to authors to listen all these kind of notifications and for this purpose we embedded Test to Speech Libraries in this layer. The new happening eventer are added into the notification list and whenever an blind author wants to listen he presses the ctrl $+p$ key to play the notification one by one as shown in Fig. 2. To listen a particular notification special short keys defined like if the author just want to know who is currently started his session the he presses ctrl $+\mathrm{p}+\mathrm{s}$.

Notifications
- Jhon started his session at
$11: 23$
- Roye Updated section 5
- Janny opened the document at
12:41
- Royc is inactive in his session
- Janny modified the figure 3 at
13:31
- Janny wants to communicate
with you
11 $m$ m $m$.

Fig. 2. Notification system's screenshot 


\section{CONCLUSION AND FUTURE WORK}

There are many application that support Web base coauthoring to produce a shared documents, but none of them have any special feature or facility that are particularly design for blind authors. In the developed system, The Amaya Thot library is used to manage and manipulate the shared documents. IIBA framework is developed to enhance awareness to keeps a context for group activities between the blind authors. Some extra feature and functions are added in the interface which is especially designed for blind. Thus, finally we are managed to develop a platform that is particularly design for blind, enhanced the group awareness among blind collaborators and inform them about all what is going on the shared environment. One of future extension of our platform is to add communication and coordination facilities for blinds. A chatting platform is added in the system by which they can communicate with other authors and get help in case of any trouble. Another extension is to make this platform useable for deaf too.

\section{REFERENCES}

[1] Preguiça N., Martins J. L., Domingues H. and Duarte S., "Data management support for asunchronous groupware," Proceedings of CSCW'00, pp. 69-78, Philadelphia, USA. ACM Press, December 2-6, 2000.

[2] Kirsch-Pinheiro, Manuele Lima, José Valdeni Borges and Marcos R.S., "Awareness em Sistemas de Groupware," Proceedings of IV Jornadas Iberoamericanas de Ingenieria de Requisitos y Ambientes de Software. Santo Domingo. Costa Rica : CIT, 2001.

[3] Fucks H. and Assis R., "Facilitating perception on virtual learning based environments," Journal of Systems and Information Technologies, Vol. 5, No. 1, pp. 93-113, Edith Cowan University, 2001.

[4] R. Baecker, D. Nastos, I. Posner and K. Mawby, "The user-centred iterative design of collaborative writing software," InterCHI'93, pp. 399405, Amsterdam, 24 - 29 April 1993.

[5] J. Begole, C. Struble, C. Shaffer and R. Smith, "Transparent sharing of Java applets: a replicated approac," 10th Annual ACM symposium on User Inledace Sofhvare and Technology, pp. 55 - 64, Alberta, Canada, 14 -17 October 1997.

[6] Y. Yang, C. Sun, Y. Zhang and X. Jia, "Real-time cooperative editing on the Internet," IEEE Internet Computing, vol. 4(1), pages 18-25, 2000.
[7] M. Locasto, M. Hulme, R. Gladysiewicz, J. Tracy and U. Wolz, "Clay: synchronous collaborative interactive environment," The Jolrnnal of Computing in Small Colleges, vol. 17(6), pp. 278-281, 2002.

[8] C. Qingzhang, H. Zangyin and Y. Kezhen, "XML-based collaborative documents model design," 8th International Conference on Computer Supported Cooperative Work in Design, vol. 1, pp. 24-28, Xiamen, China, 2003.

[9] M. Weber, G. Partsch, S. Hock, G. Schneider, A. Scheller-Houy and J. Schweitzer, "Integrating synchronous multimedia collaboration into workflow management," International ACM SIGGROUP Conference on Supporting Group Work, pp. 28 1-290, Phoenix, Arizona, 16-19 November 1997.

[10] T. Hodel, H. Gall and K. Dittrich, "Dynamic collaborative business processes within documents," SZGDOC 2004, pp. 97-103, Memphis, USA, 10 - 13 October 2004.

[11] G. Joeris, "Cooperative and integrated workflow and document management for engineering applications," Eighth International Workshop on Database and Expert Systems Applications, pp. 68-73, Toulouse, France, 1-2 September 1997.

[12] K. Ho, H. Leong and W. Lam, "A collaborative word processing system using a CORBA-based workflow system," 3rd International Symposium on Distributed Objects \& Applications, pp. 176- 185, Rome, Italy, 17-20 September 2001.

[13] http://www.who.int/mediacentre/factsheets/en/, last visited $10 \mathrm{Apr}, 2015$

[14] T. Dasgupta, and A. Basu, "A speech enabled Indian language text to Braille transliteration system," information and Communication Technologies and Development (ICTD), pp-201- 211, 2009.

[15] N. Kalra, T. Lauwers, D. Dewey, T. Stepleton and M. B. Dias, "Iterative design of a Braille writing tutor to combat illiteracy," Proceedings of the 2nd IEEE/ACM International Conference on Information and Communication Technologies and Development, December, 2007.

[16] Aslam Muhammad, A.M. Martinez and G. Escalada-Imz, "Collaborative Assistant to Handel MathML Expressions," Calculemus/MKM 2009, LNAI 5625, pp. 453-459, 2009.

[17] Leventhal, J., "Not What the Doctor Ordered: A Review of Apple's VoiceOver Screen Reader." 2005.

[18] Sonia Mendoza-Chapa, Manuel Romero-Salcedo, Hanna Oktaba, "Group Awareness Support in Collaborative Writing Systems," 2000.

[19] R. Guetari, V. Quint and I. Vatton, "Amaya: an Authroing Tool for Web," in proc. MCSEAI'98 Maghrebian Conference on Software Engineering and Artificial Intelligence, Tunis, Tunisia, 8-10 Descember 1998. 